\title{
Supplementary Material for Synthesis of Compact Multidentate Ligands to Prepare Stable Hydrophilic Quantum Dot Fluorophores
}

5-[1,2]-Dithiolan-3-yl-pentanoic acid 2-[2-(2-\{2-[2-(2-hydroxy-ethoxy)-ethoxy]-ethoxy\}ethoxy)-ethoxy]-ethyl ester [TA-HEG]: Compound (2). Compound 2 was prepared from thioctic acid and hexaethylene glycol following the procedure used for compound 1. Yield: 53\% as a yellow oil. TLC (EtOAc/MeOH 95:5) $R_{f} 0.35 ;{ }^{1} \mathrm{H}-\mathrm{NMR}\left(400 \mathrm{MHz}, \mathrm{CDCl}_{3}\right): \delta(\mathrm{ppm}) 4.19(\mathrm{t}$, $J=4.8 \mathrm{~Hz}, 2 \mathrm{H}), 3.69-3.51(\mathrm{~m}, 22 \mathrm{H}), 3.13(\mathrm{~m}, 2 \mathrm{H}), 2.76(\mathrm{~s}, 1 \mathrm{H}), 2.43(\mathrm{~s}, 1 \mathrm{H}), 2.32(\mathrm{t} J=7.5$ $\mathrm{Hz}, 2 \mathrm{H}), 1.88(\mathrm{~m}, 1 \mathrm{H}), 1.62(\mathrm{~m}, 4 \mathrm{H}), 1.43(\mathrm{~m}, 2 \mathrm{H}) .{ }^{13} \mathrm{C} \mathrm{NMR}\left(100 \mathrm{MHz}, \mathrm{CDCl}_{3}\right): \delta(\mathrm{ppm})$ $172.76,72.10,69.99,69.92,69.68,68.53,62.87,60.95,55.73,39.65,37.92,34.00,33.33,28.11$, 24.03.

[TA-PEG 400]: Compound (3). Compound 3 was prepared from thioctic acid and poly(ethylene glycol) $400 \mathrm{MW}$ following the procedure used for compound 1. Yield: $75 \%$ as a yellow oil. TLC $\left(\mathrm{CHCl}_{3} / \mathrm{MeOH} /\right.$ acetone 90:5:5) $R_{f} 0.3 ;{ }^{1} \mathrm{H}-\mathrm{NMR}\left(400 \mathrm{MHz}, \mathrm{CDCl}_{3}\right): \delta(\mathrm{ppm})$ $4.09(\mathrm{t}, J=4.8 \mathrm{~Hz}, 2 \mathrm{H}), 3.52(\mathrm{~m}, 36 \mathrm{H}), 3.02(\mathrm{~m}, 2 \mathrm{H}), 2.52(\mathrm{~s}, 1 \mathrm{H}), 2.33(\mathrm{~m}, 1 \mathrm{H}), 2.22(\mathrm{t}, J=7.4$ $\mathrm{Hz}, 2 \mathrm{H}), 1.79(\mathrm{~m}, 1 \mathrm{H}), 1.54(\mathrm{~m}, 4 \mathrm{H}), 1.34(\mathrm{~m}, 2 \mathrm{H}) .{ }^{13} \mathrm{C} \mathrm{NMR}\left(100 \mathrm{MHz}, \mathrm{CDCl}_{3}\right): \delta(\mathrm{ppm})$ $173.06,72.26,70.16,69.89,68.74,63.08,61.21,55.93,49.94,39.83,38.10,34.19,33.53,28.31$, 24.22.

[TA-PEG 600]: Compound (4). Compound 4 was prepared from thioctic acid and poly(ethylene glycol) $600 \mathrm{MW}$ following the procedure used for compound 1. Yield: $75 \%$ as a yellow oil. TLC $\left(\mathrm{CHCl}_{3} / \mathrm{MeOH} /\right.$ acetone $\left.90: 5: 5\right) R_{f} 0.3 ;{ }^{1} \mathrm{H}-\mathrm{NMR}\left(400 \mathrm{MHz}, \mathrm{CDCl}_{3}\right): \delta(\mathrm{ppm})$ $4.18(\mathrm{t}, J=4.8 \mathrm{~Hz}, 2 \mathrm{H}), 3.7-3.5(\mathrm{~m}, \sim 50 \mathrm{H}), 3.11(\mathrm{~m}, 2 \mathrm{H}), 2.58(\mathrm{br} \mathrm{s}, 1 \mathrm{H}), 2.43(\mathrm{~m}, 1 \mathrm{H}), 2.31$ $(\mathrm{t}, J=7.4 \mathrm{~Hz}, 2 \mathrm{H}), 1.87(\mathrm{~m}, 1 \mathrm{H}), 1.7-1.55(\mathrm{~m}, 4 \mathrm{H}), 1.5-1.35(\mathrm{~m}, 2 \mathrm{H}){ }^{13} \mathrm{C} \mathrm{NMR}(100 \mathrm{MHz}$, 
$\left.\mathrm{CDCl}_{3}\right): \delta(\mathrm{ppm}) 172.83,72.10,70.08,69.87,68.64,62.96,61.12,55.81,39.73,38.00,34.10$ $33.43,28.21,24.13$.

[TA-PEG-1000]: Compound (5). Compound 5 was prepared from thioctic acid and poly(ethylene glycol) $1000 \mathrm{MW}$ following the procedure used for compound $\mathbf{1}$. The reaction provides a yellow waxy solid with a yield of $\sim 52 \%$. TLC $\left(\mathrm{CHCl}_{3} / \mathrm{MeOH} 9: 1\right) R_{f} 0.4 ;{ }^{1} \mathrm{H}-\mathrm{NMR}$ (400 MHz, $\left.\mathrm{CDCl}_{3}\right): \delta(\mathrm{ppm}) 4.15(\mathrm{t}, J=4.7 \mathrm{~Hz}, 2 \mathrm{H}), 3.7-3.5(\mathrm{~m}, \sim 90 \mathrm{H}), 3.14(\mathrm{~m}, 2 \mathrm{H}), 2.50(\mathrm{br}$ s, 1H), $2.44(\mathrm{~m}, 1 \mathrm{H}), 2.33(\mathrm{t}, J=7.4 \mathrm{~Hz}, 2 \mathrm{H}), 1.85(\mathrm{~m}, 1 \mathrm{H}), 1.75-1.5(\mathrm{~m}, 4 \mathrm{H}), 1.4-1.3(\mathrm{~m}$, 2H). ${ }^{13} \mathrm{C}$ NMR (100 MHz, $\left.\mathrm{CDCl}_{3}\right): \delta(\mathrm{ppm}) 173.08,72.30,70.20,69.93,68.79,63.11,61.25$, $55.96,50.01,39.87,38.13,34.23,33.58,28.35,24.26$.

\section{6,8-Dimercapto-octanoic acid 2-[2-(2-\{2-[2-(2-hydroxy-ethoxy)-ethoxy]-ethoxy\}-ethoxy)-} ethoxy]-ethyl ester [DHLA-HEG]: Compound (8). Compound 8 was prepared according to the above general procedure described for compound 7. It yields a colorless oil with $\sim 85 \%$ efficiency. TLC (EtOAc/MeOH 9:1) $R_{f} 0.4 ;{ }^{1} \mathrm{H}-\mathrm{NMR}\left(400 \mathrm{MHz}, \mathrm{CDCl}_{3}\right): \delta(\mathrm{ppm}) 4.19$ (t, $J=$ $4.8 \mathrm{~Hz}, 2 \mathrm{H}), 3.65(\mathrm{~m}, 20 \mathrm{H}), 3.57(\mathrm{t}, J=4.5 \mathrm{~Hz}, 2 \mathrm{H}), 2.88(\mathrm{~m}, 1 \mathrm{H}), 2.67(\mathrm{~m}, 3 \mathrm{H}), 2.32(\mathrm{t}, J=7.3$ $\mathrm{Hz}, 2 \mathrm{H}), 1.87(\mathrm{~m}, 1 \mathrm{H}), 1.75-1.38(\mathrm{~m}, 7 \mathrm{H}), 1.32(\mathrm{t}, J=8.0 \mathrm{~Hz}, 1 \mathrm{H}), 1.27(\mathrm{~d}, J=7.7 \mathrm{~Hz}, 1 \mathrm{H})$.

${ }^{13} \mathrm{C}$ NMR $\left(100 \mathrm{MHz}, \mathrm{CDCl}_{3}\right): \delta(\mathrm{ppm}) 173.11,72.29,70.22,70.16,69.90,68.79,63.11,61.23$, $42.40,38.95,38.34,33.63,26.13,24.17,21.93$.

[DHLA-PEG 400]: Compound (9). Compound 9 was also prepared according to the above general procedure for 7 . The procedure has a yield of $\sim 75 \%$ and provides a colorless oil. TLC $\left(\mathrm{CHCl}_{3} / \mathrm{MeOH} 95: 5\right) R_{f} 0.25 ;{ }^{1} \mathrm{H}-\mathrm{NMR}\left(400 \mathrm{MHz}, \mathrm{CDCl}_{3}\right): \delta(\mathrm{ppm})$ 
[DHLA-PEG 600]: Compound (10). Compound 10 was also prepared according to the above general procedure for 7 . The procedure has a yield of $\sim 93 \%$ and provides a colorless oil. TLC $\left(\mathrm{CHCl}_{3} / \mathrm{MeOH} /\right.$ acetone 90:5:5) $R_{f} 0.25 ;{ }^{1} \mathrm{H}-\mathrm{NMR}\left(400 \mathrm{MHz}, \mathrm{CDCl}_{3}\right): \delta(\mathrm{ppm}) 4.18(\mathrm{t}, J=4.8 \mathrm{~Hz}$, 2H), $3.62(\mathrm{~m}, \sim 50 \mathrm{H}), 2.88(\mathrm{~m}, 1 \mathrm{H}), 2.66(\mathrm{~m}, 3 \mathrm{H}), 2.31(\mathrm{t}, J=7.3 \mathrm{~Hz}, 2 \mathrm{H}), 1.85(\mathrm{~m}, 1 \mathrm{H}), 1.75-$ $1.35(\mathrm{~m}, 7 \mathrm{H}), 1.31(\mathrm{t}, J=8.0 \mathrm{~Hz}, 1 \mathrm{H}), 1.26(\mathrm{~d}, J=7.6 \mathrm{~Hz}, 1 \mathrm{H}) .{ }^{13} \mathrm{C} \mathrm{NMR}\left(100 \mathrm{MHz}, \mathrm{CDCl}_{3}\right)$ : $\delta(\mathrm{ppm}) 173.20,72.37,70.31,70.05,68.91,63.21,61.38,42.51,39.04,38.47,33.74,26.24$ $24.27,22.02$.

[DHLA-PEG-1000]: Compound (11). The general procedure described above for compound 6 was employed to prepare compound 7. The final product was a colorless waxy solid with $83 \%$ yield. TLC $\left(\mathrm{CHCl}_{3} / \mathrm{MeOH}\right.$ 9:1) $R_{f} 0.4 ;{ }^{1} \mathrm{H}-\mathrm{NMR}\left(400 \mathrm{MHz}, \mathrm{CDCl}_{3}\right): \delta(\mathrm{ppm}) 4.05$ (t, $J=4.8 \mathrm{~Hz}$, 2H), $3.47(\mathrm{~m}, \sim 90 \mathrm{H}), 2.90(\mathrm{~s}, 1 \mathrm{H}), 2.75(\mathrm{~m}, 1 \mathrm{H}), 2.52(\mathrm{~m}, 2 \mathrm{H}), 2.19(\mathrm{t}, J=7.4 \mathrm{~Hz}, 2 \mathrm{H}), 1.75(\mathrm{~m}$, 1H), $1.62-1.24(\mathrm{~m}, 7 \mathrm{H}), 1.22(\mathrm{t}, J=8.0 \mathrm{~Hz}, 1 \mathrm{H}), 1.70(\mathrm{~d}, J=7.6 \mathrm{~Hz}, 1 \mathrm{H}) .{ }^{13} \mathrm{C}$ NMR $(100$ $\left.\mathrm{MHz}, \mathrm{CDCl}_{3}\right): \delta(\mathrm{ppm}) 172.84,72.07,70.05,69.84,68.63,62.92,61.07,42.24,38.75,38.19$, 33.46, 25.96, 24.00, 21.74 . 
Table S1. Chart of solubility and representative solvents for dispersion of QDs coated with the following ligands.

\begin{tabular}{|l|l|l|l|}
\hline Capping Ligand: & \multicolumn{1}{|c|}{ TOP/TOPO } & \multicolumn{1}{c|}{ DHLA } & \multicolumn{1}{c|}{ DHLA-PEG } \\
\hline Solubility & Non-polar & Polar & Amphiphilic \\
\hline Representative & $\begin{array}{l}\text { Hexane, } \\
\text { Solvents }\end{array}$ & Basic buffers & Both acidic and basic \\
& & $\begin{array}{l}\text { methanol, ethanol, } \\
\text { dimethylformamide, } \\
\text { dimethylsulfoxide } \\
\text { dimethylformamide, } \\
\text { dimethylsulfoxide, }\end{array}$ \\
& & $\begin{array}{l}\text { dimol, ethanol, } \\
\text { chloroform }\end{array}$ \\
\hline
\end{tabular}

\section{References:}

(1) Gunsalus, I. C.; Barton, L. S.; Gruber, W. J. Am. Chem. Soc. 1956, 78, 1763-1766.

(2) Wagner, A. F.; Walton, E.; Boxer, G. E.; Pruss, M. P.; Holly, F. W.; Folkers, K. J. Am. Chem. Soc. 1956, 78, 5079-5081. 


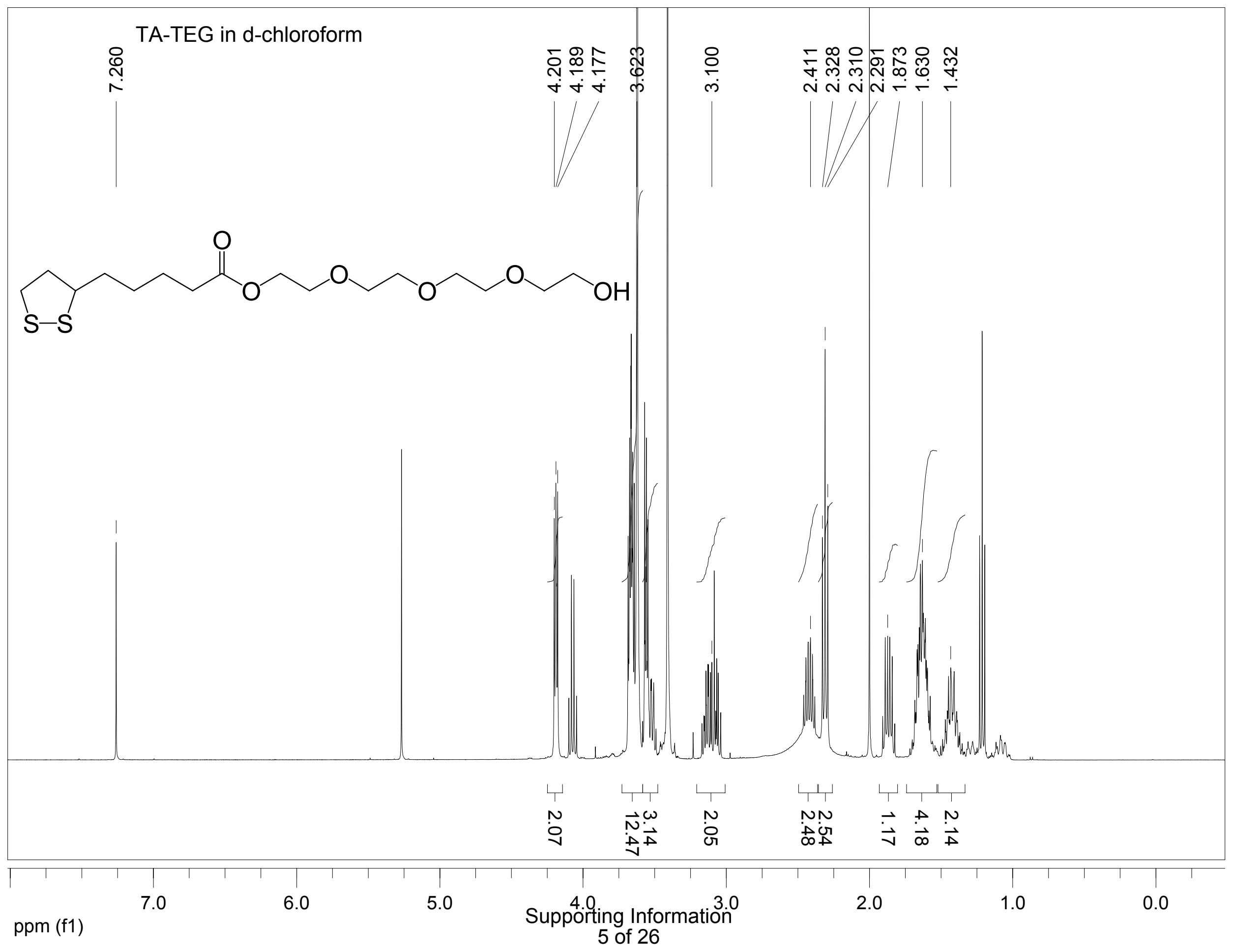




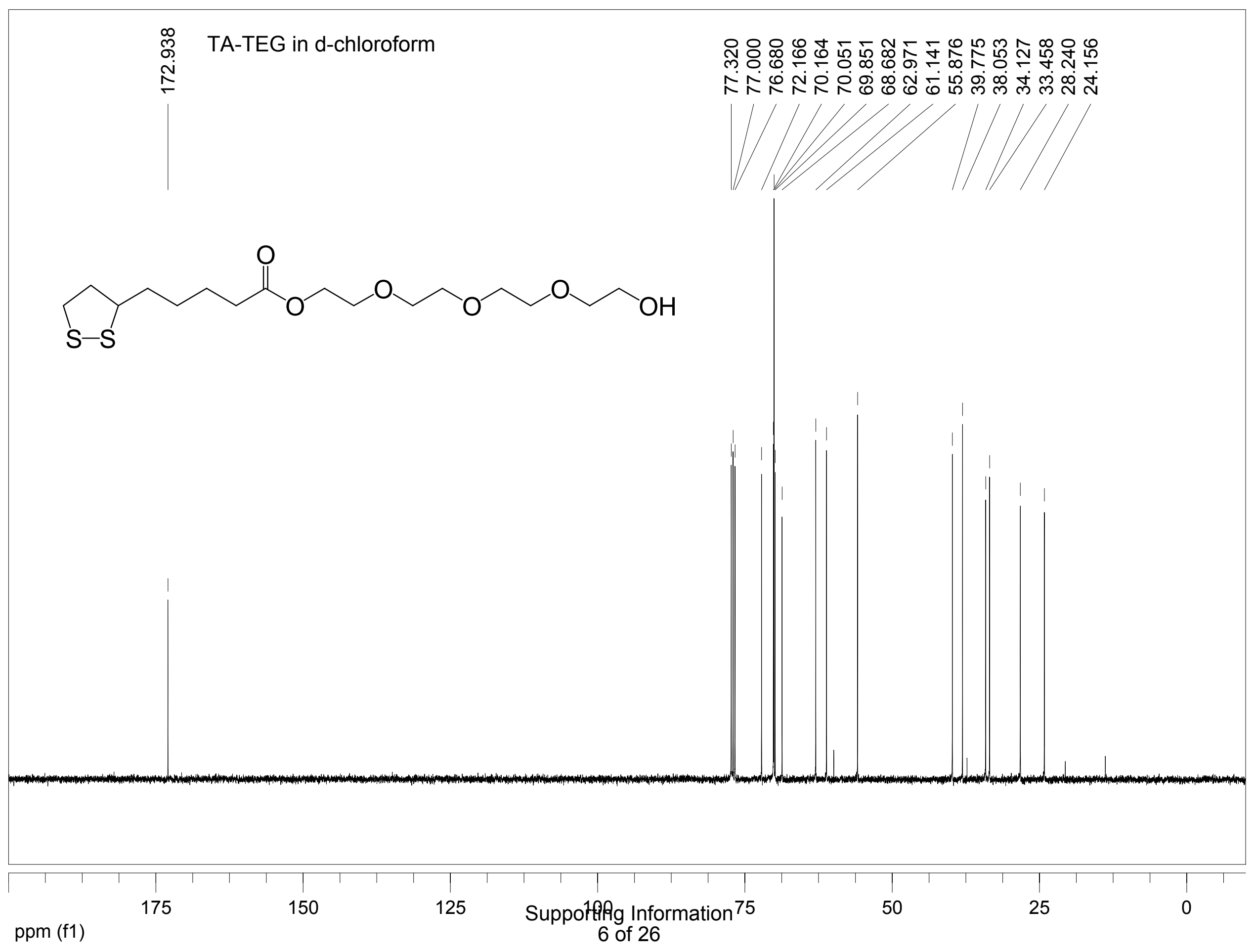




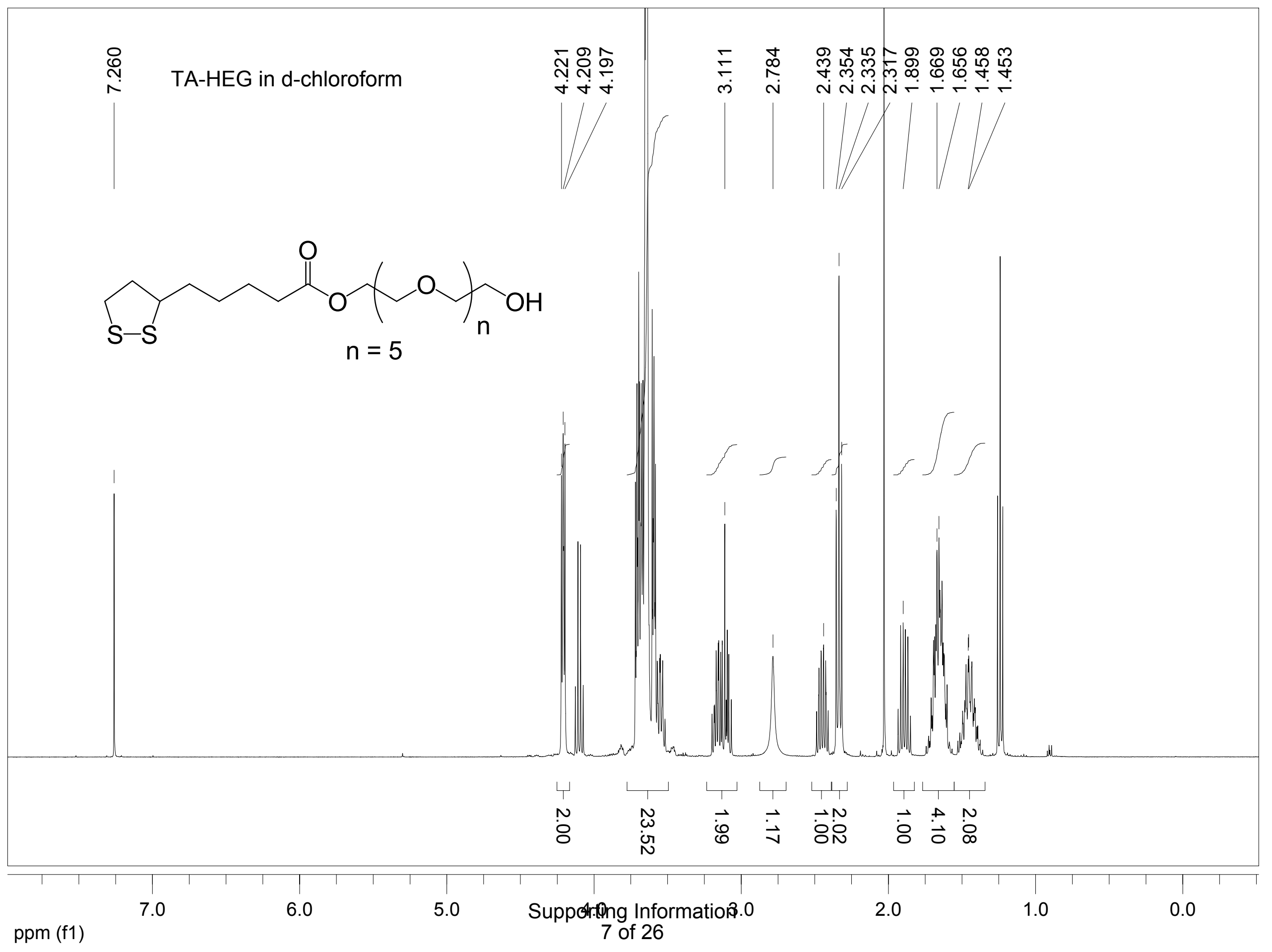




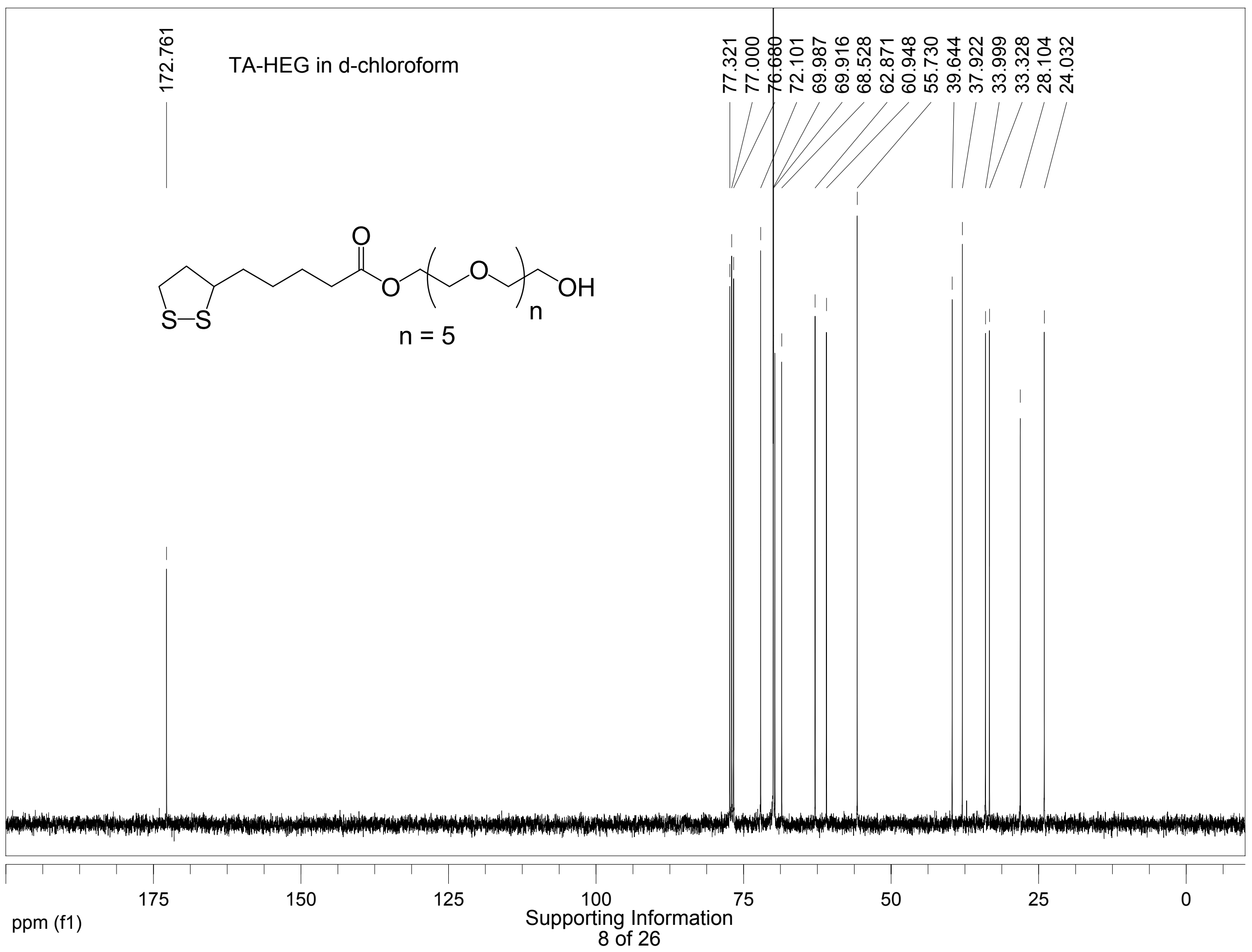




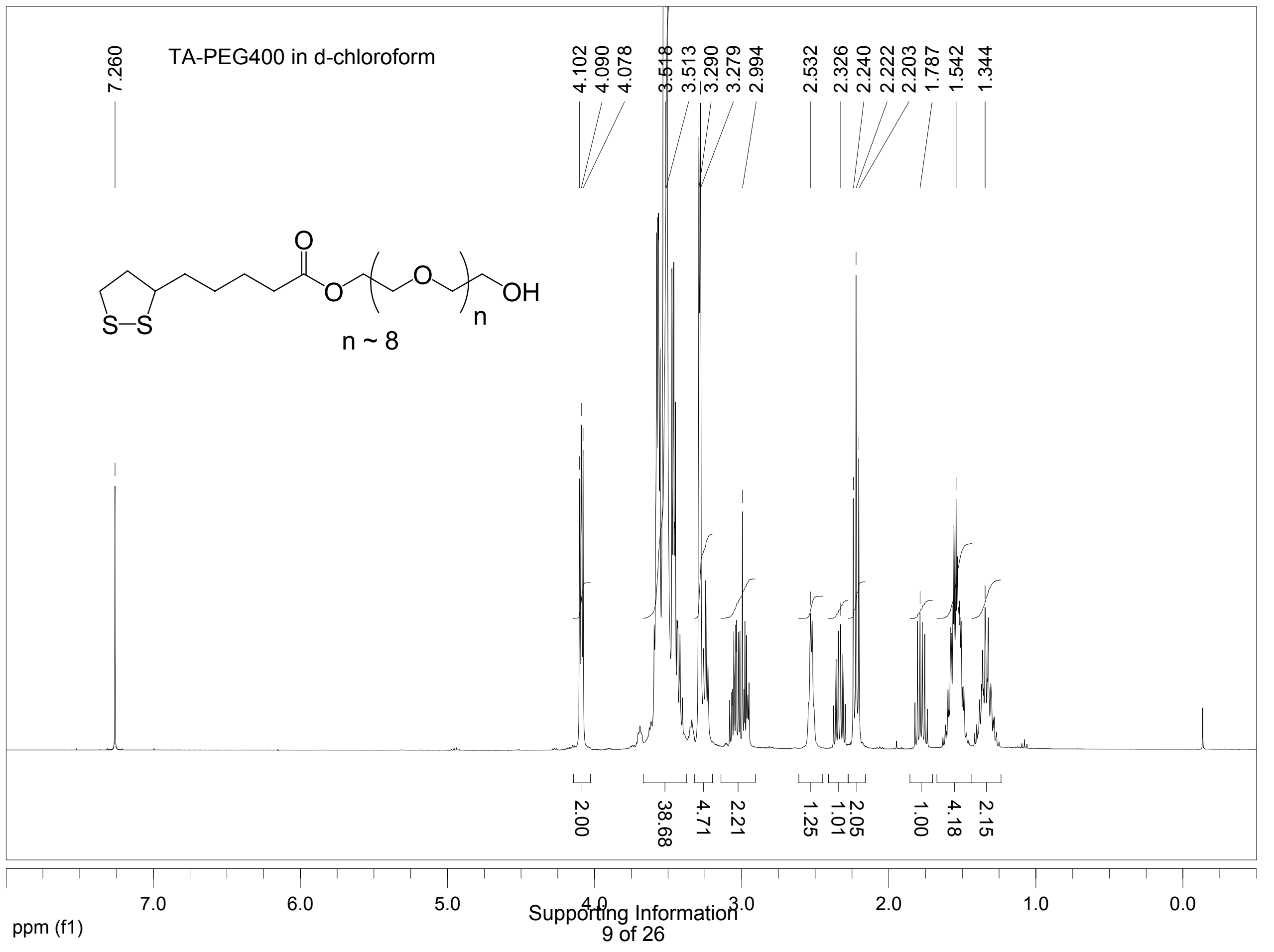




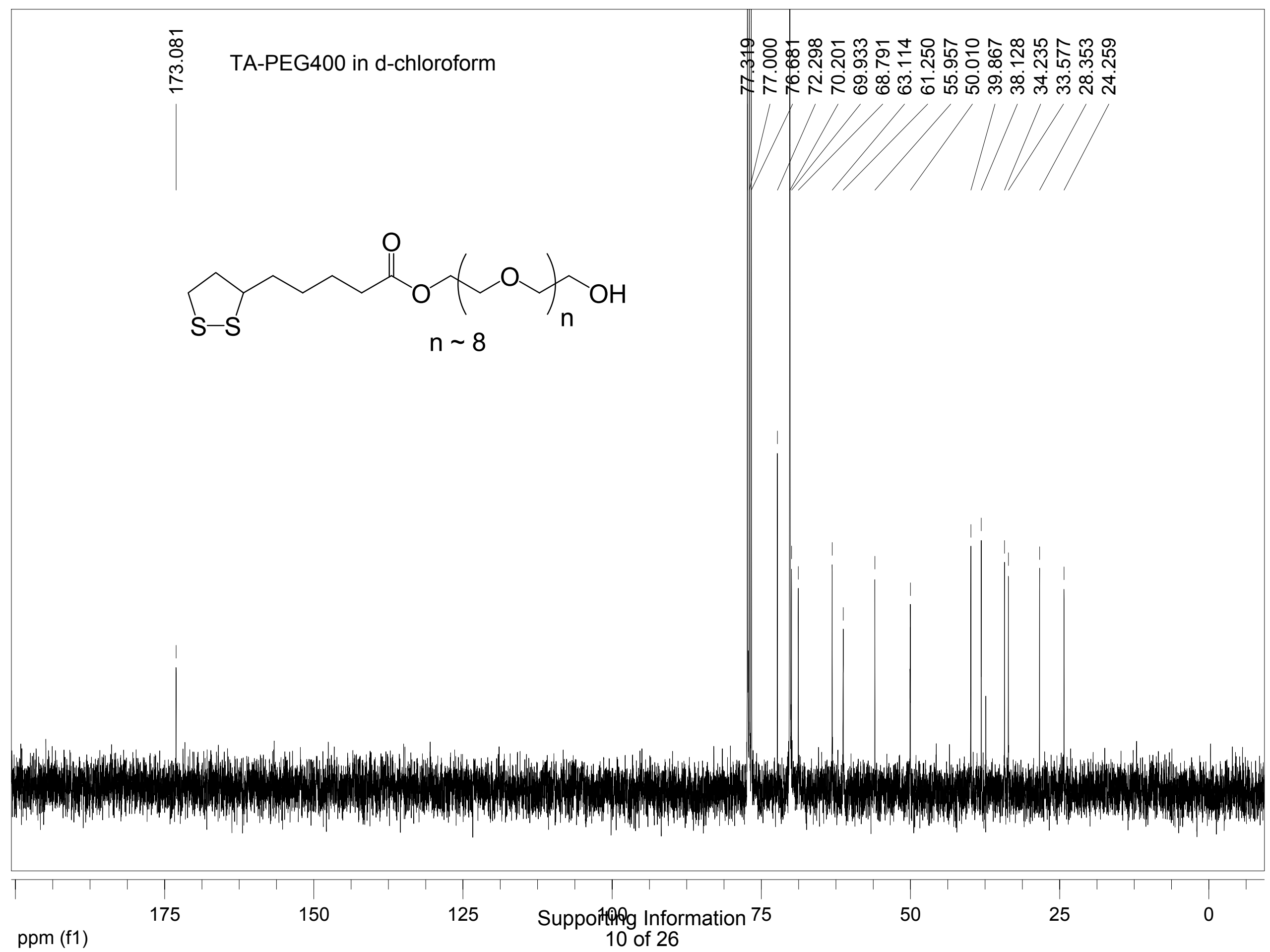




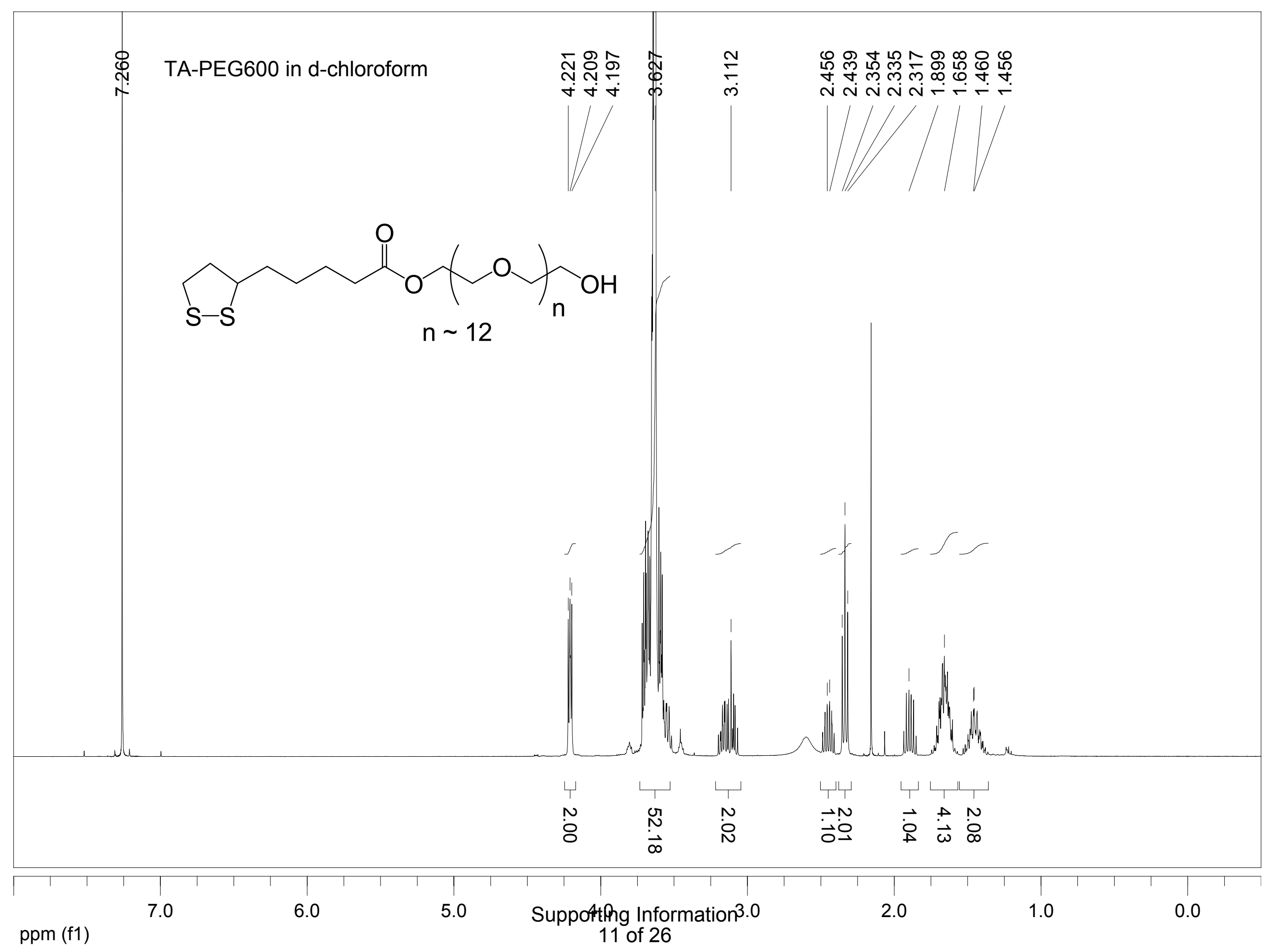




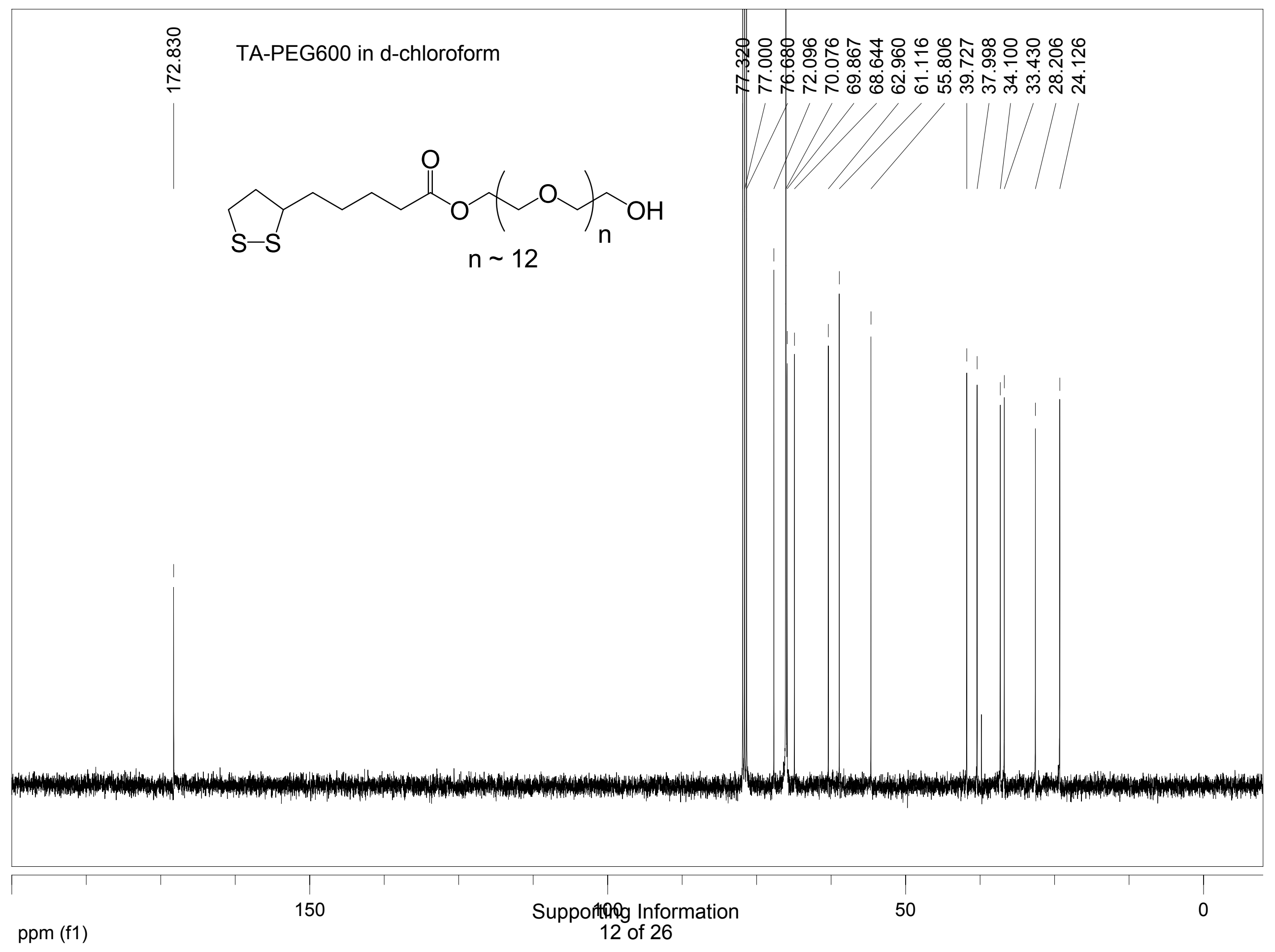




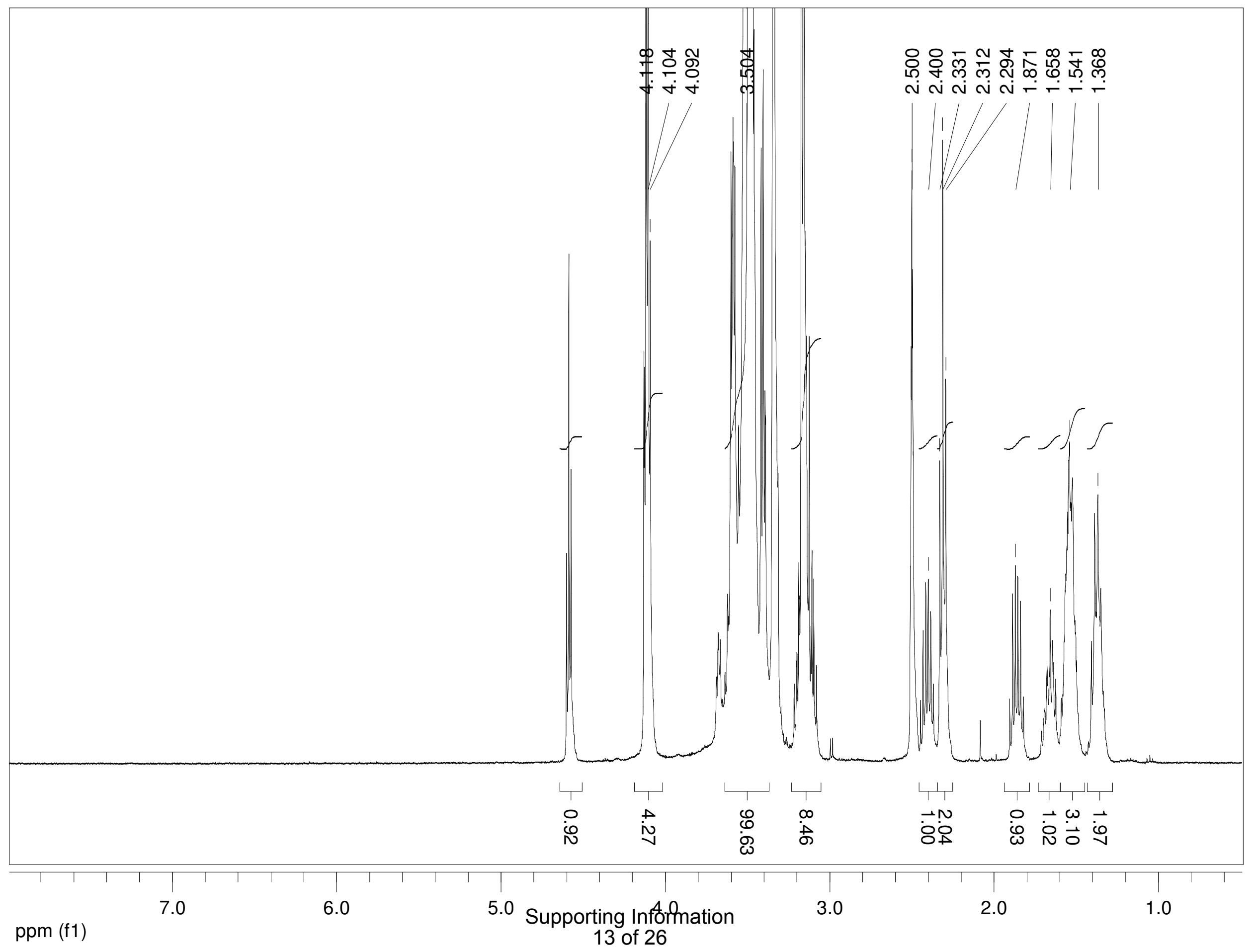




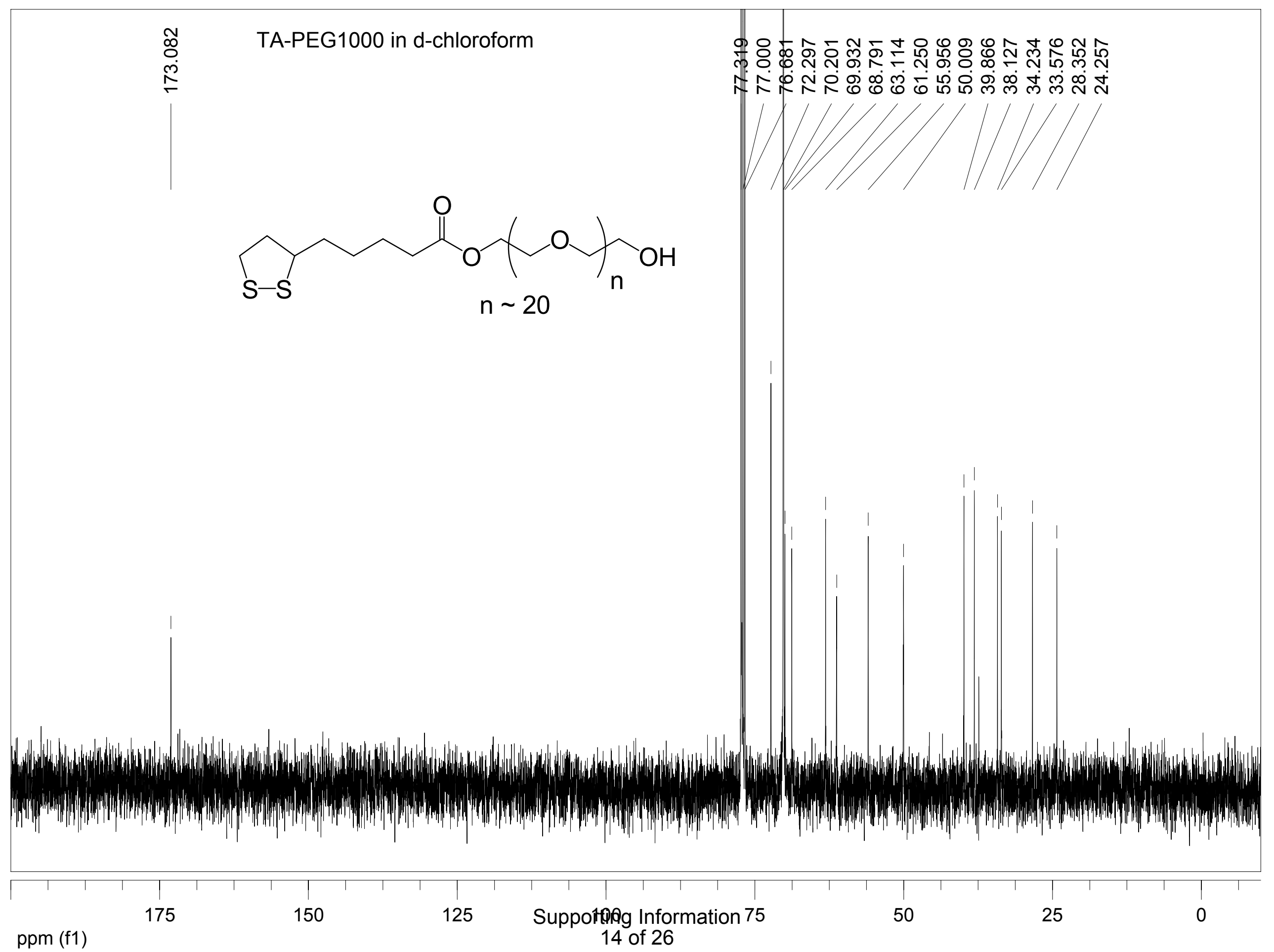



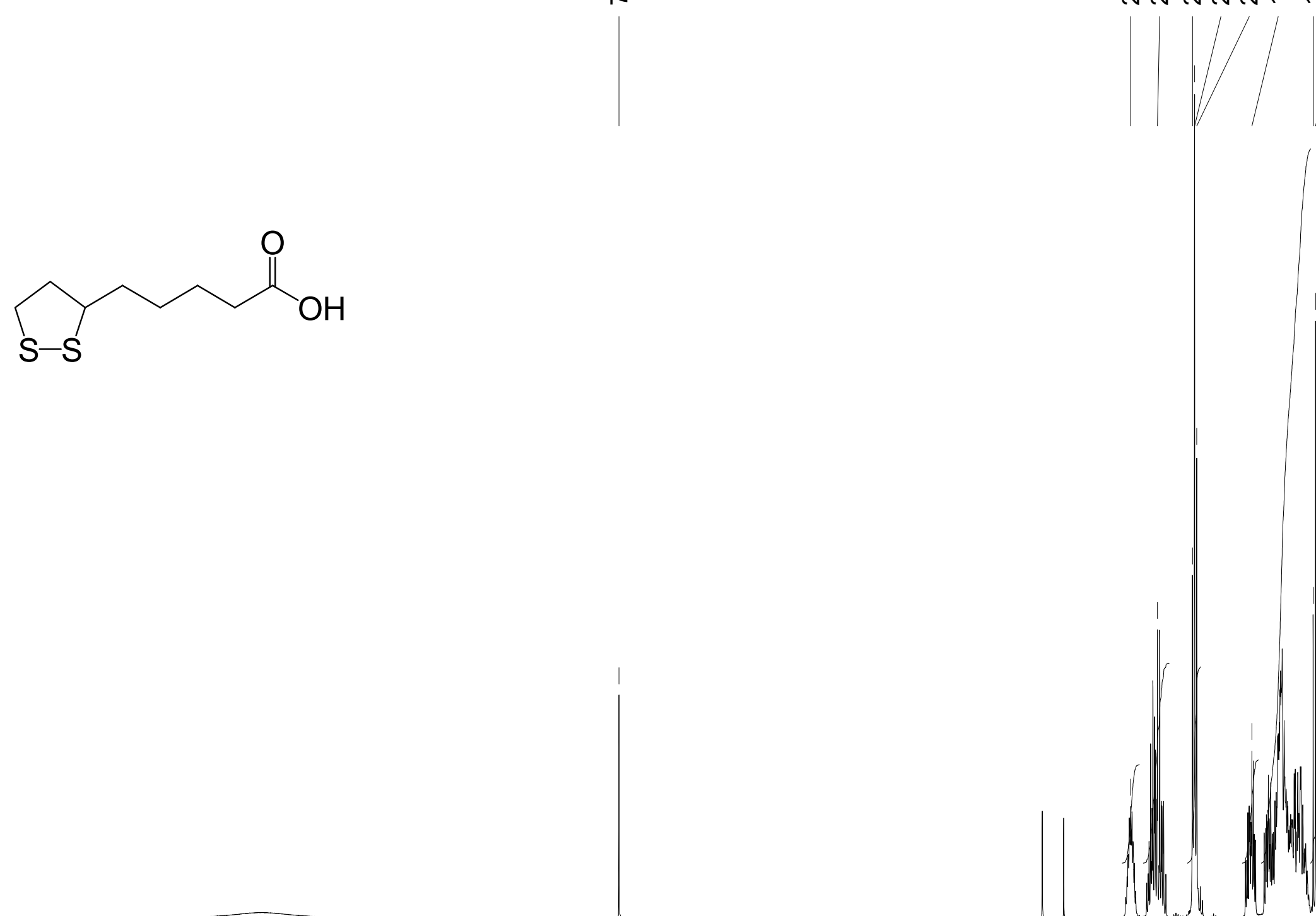

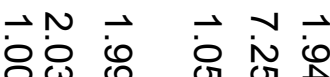

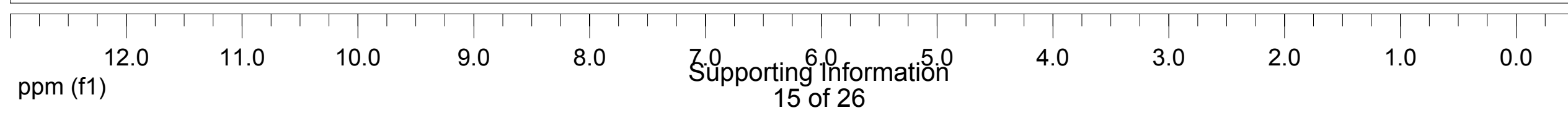




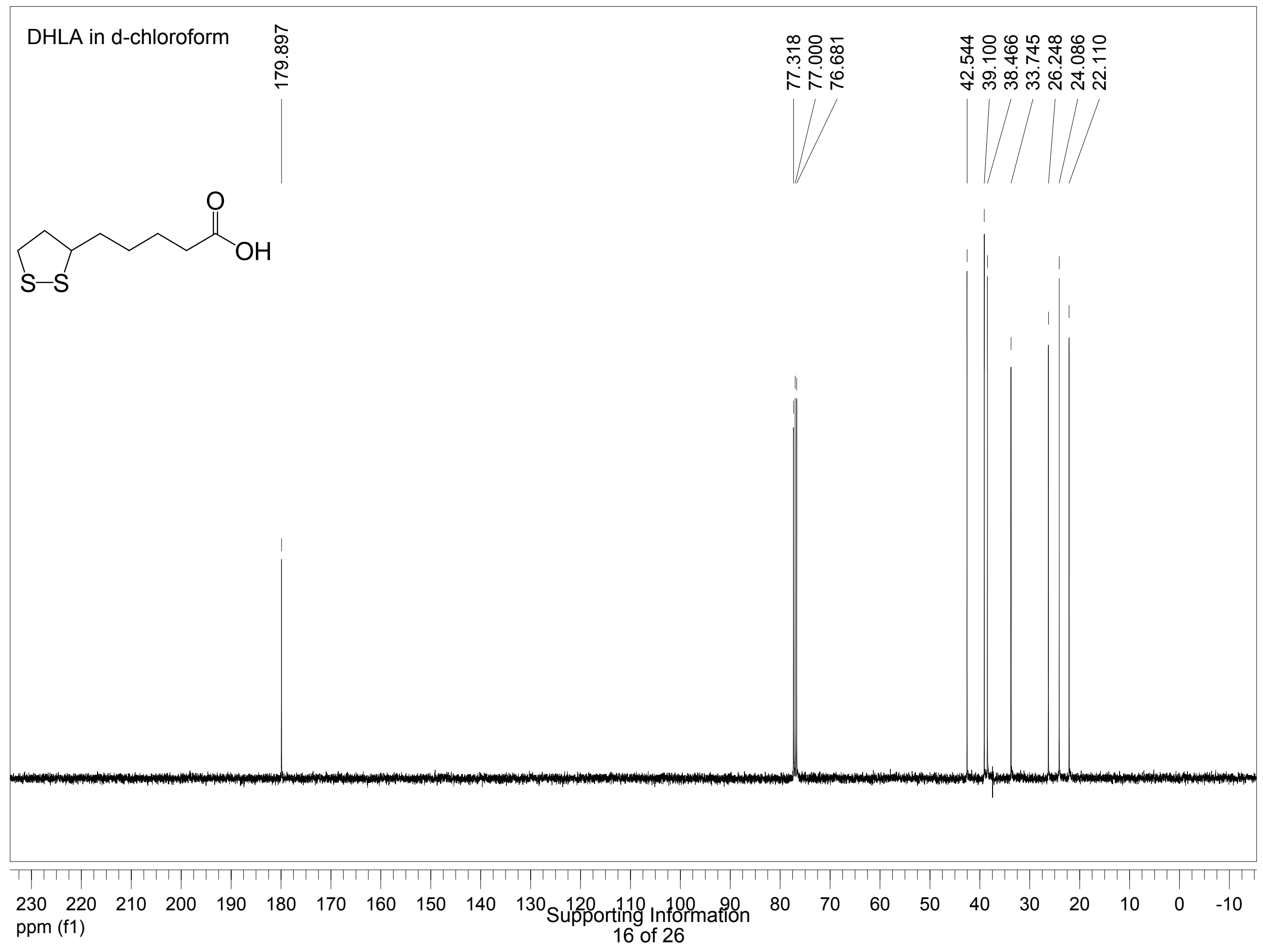




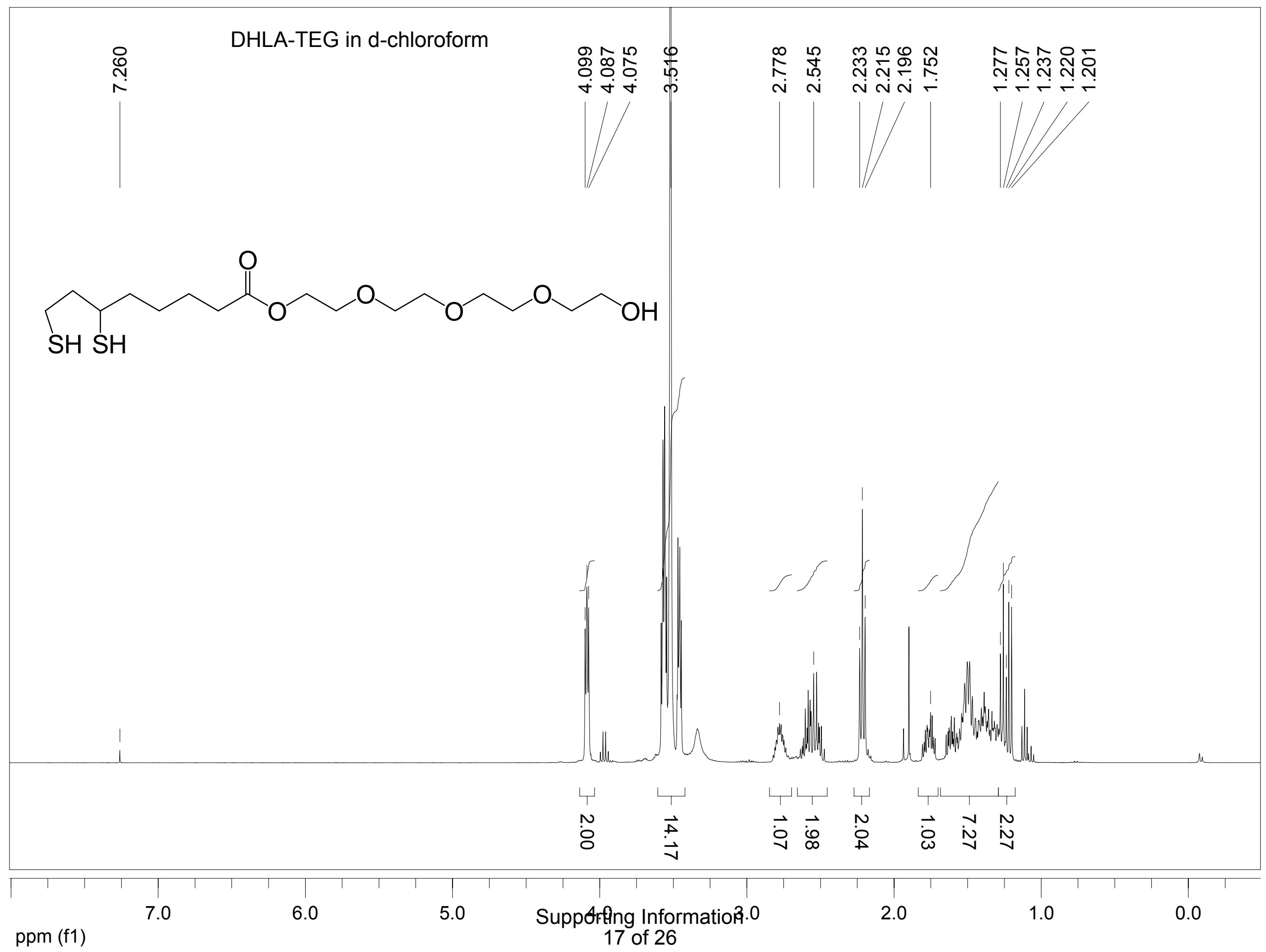




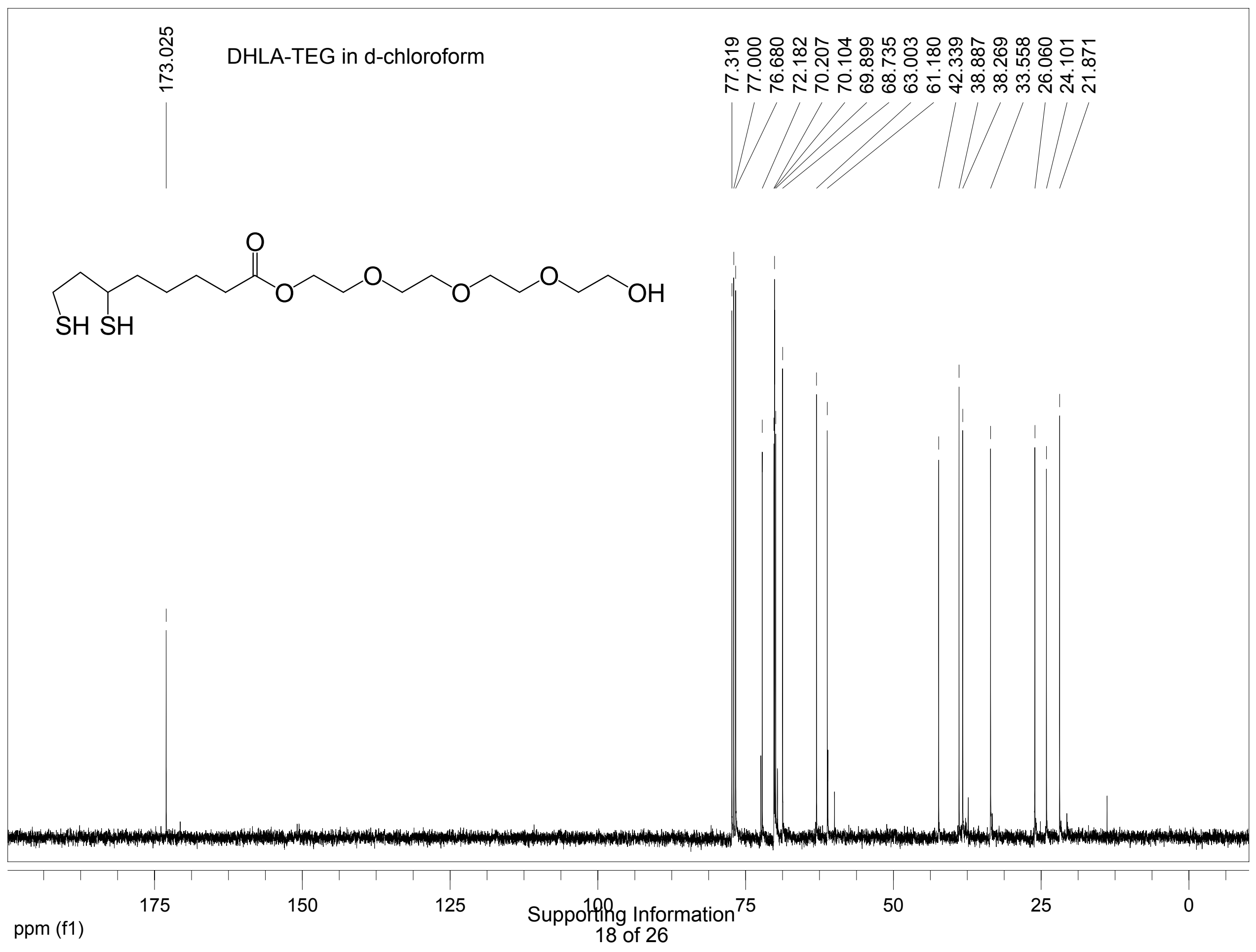




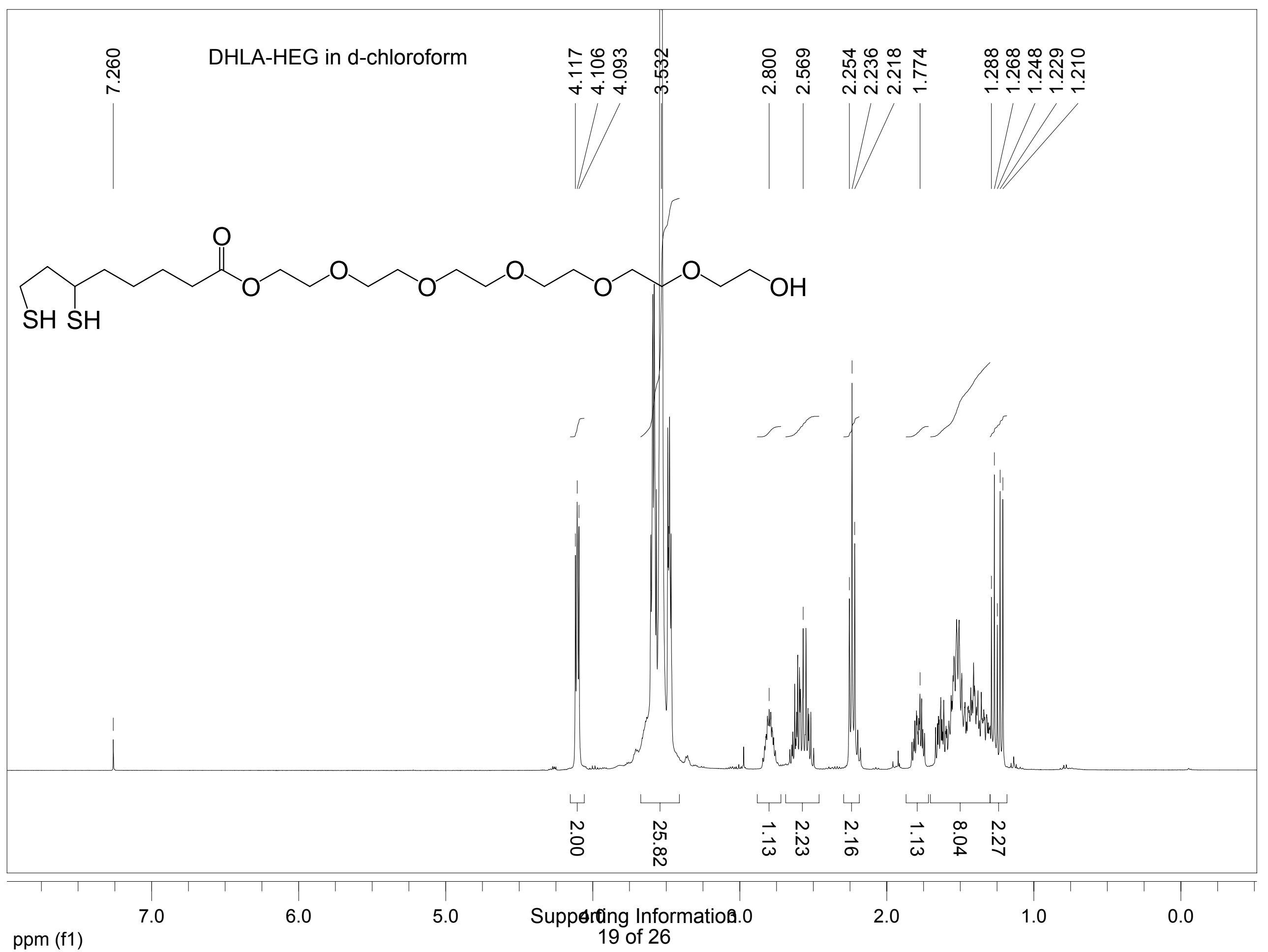




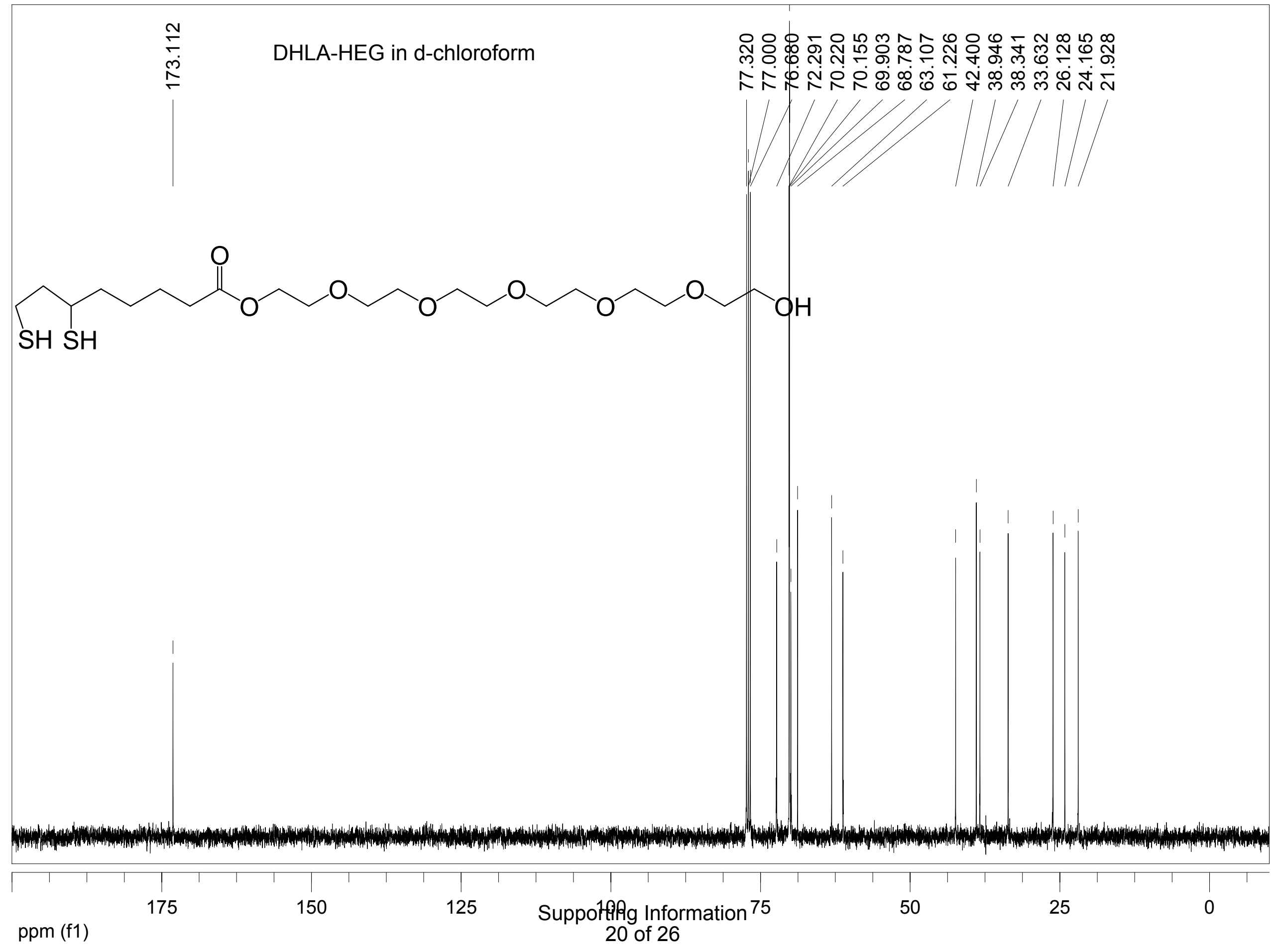




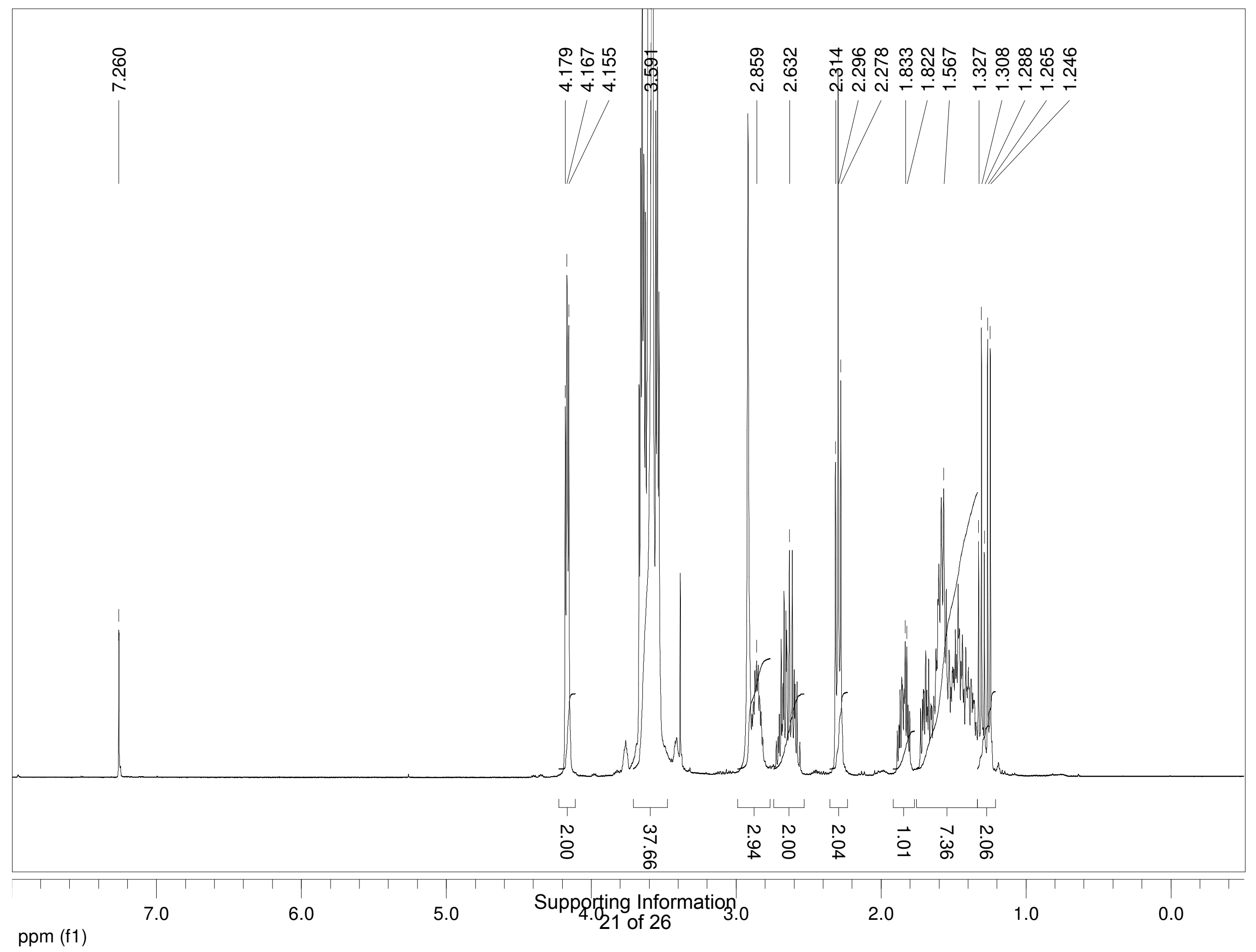




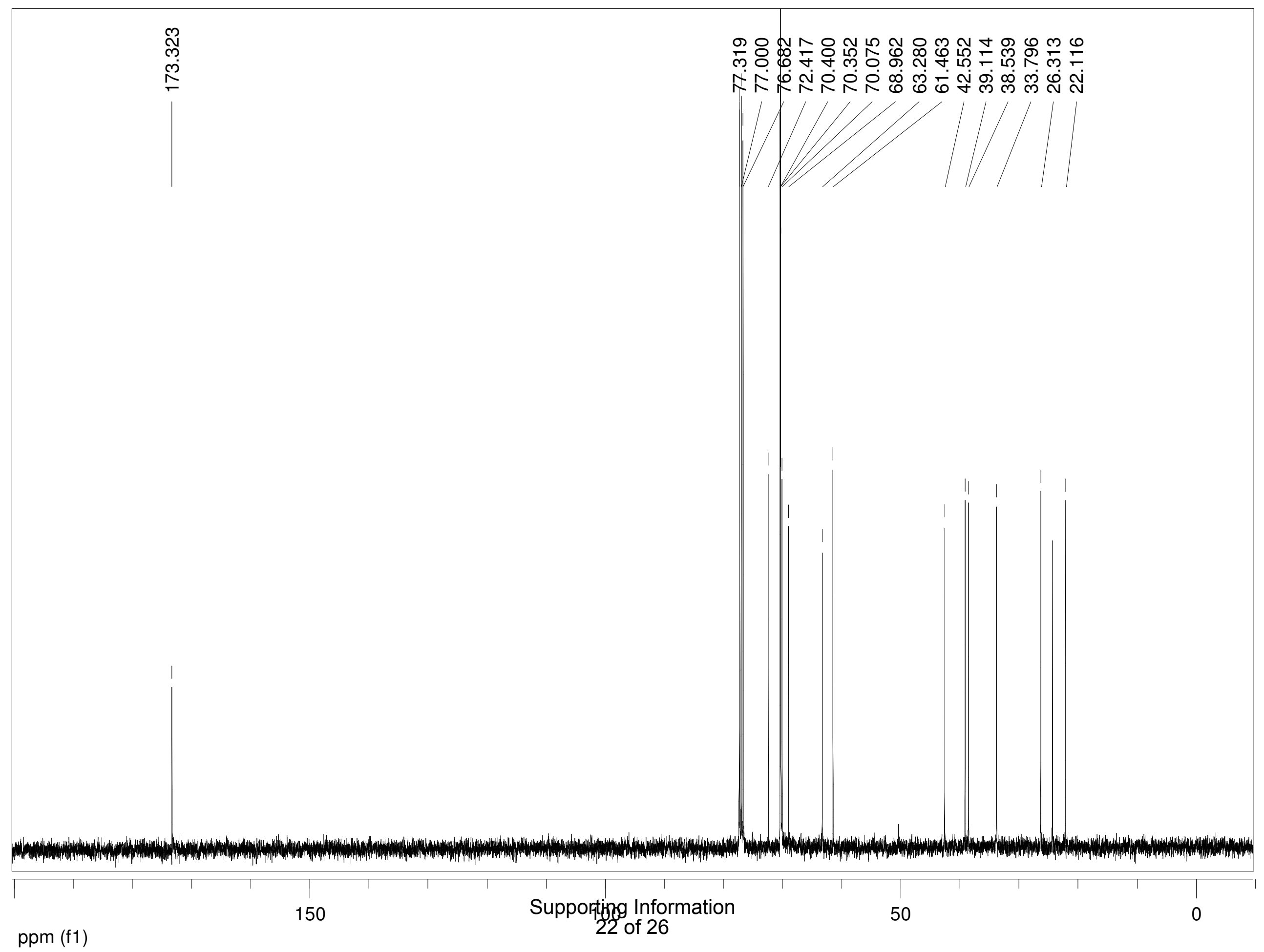




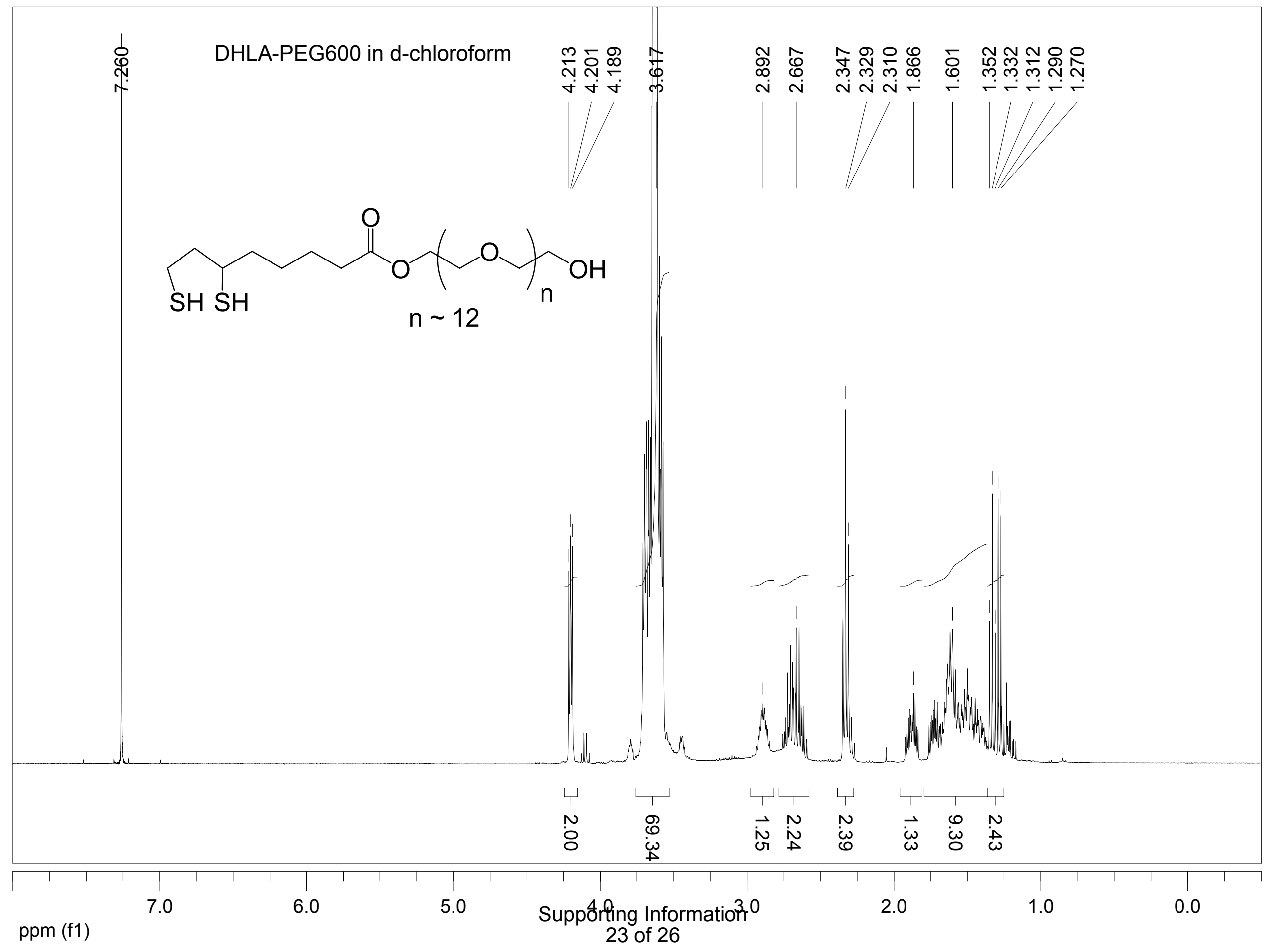




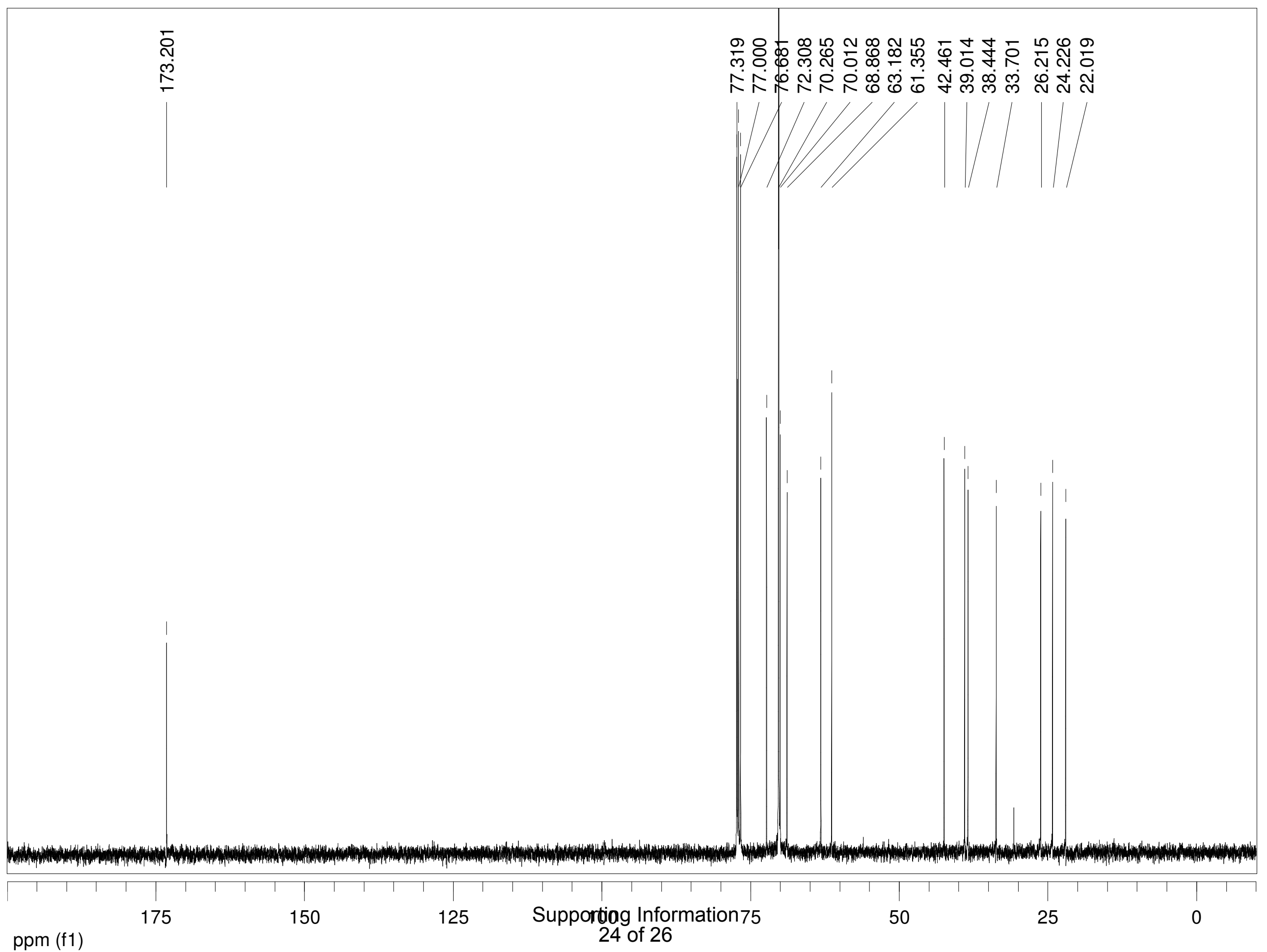




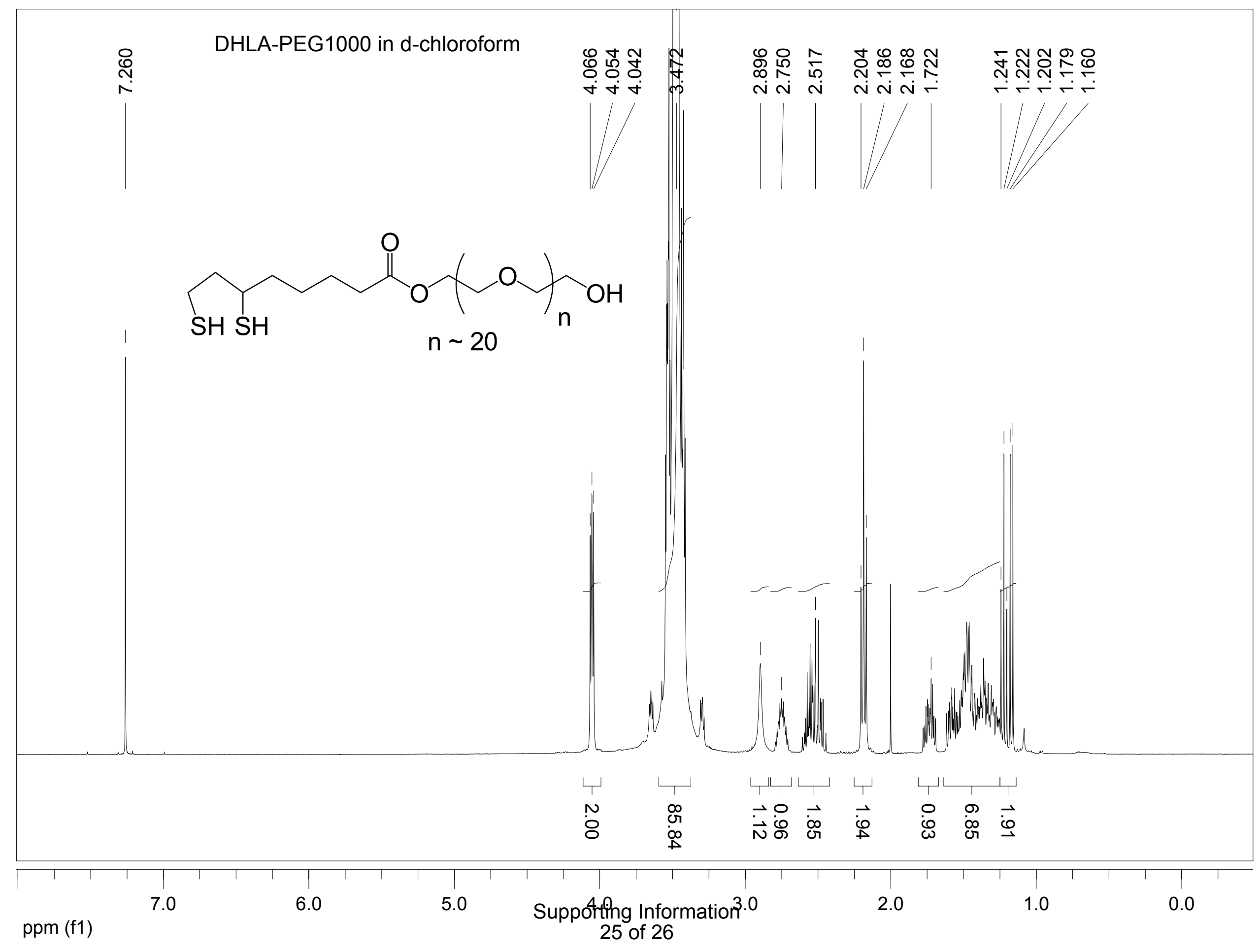




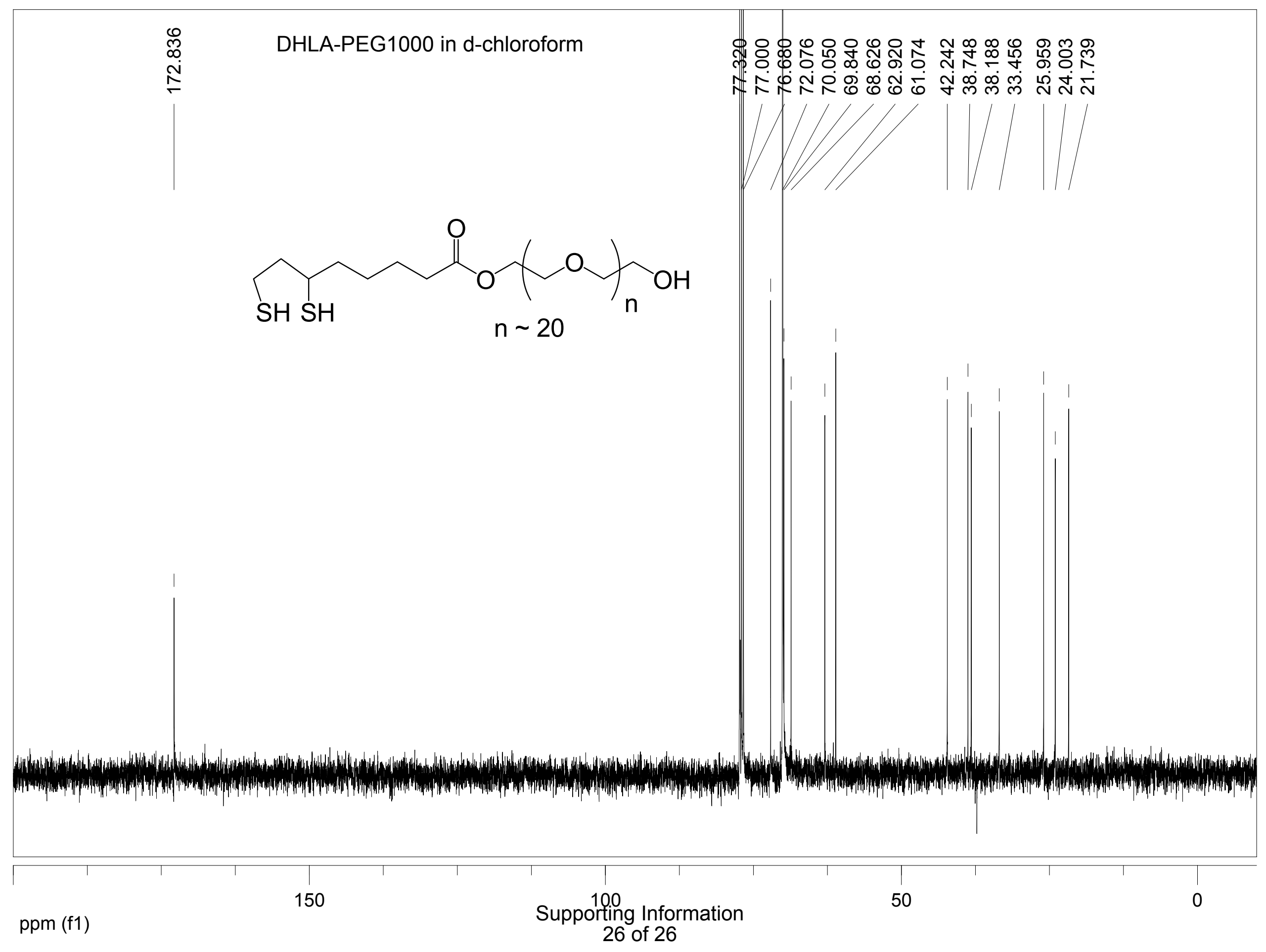

